Copyright (C 2014 IEEE. Personal use of this material is permitted. Permission from IEEE must be obtained for all other uses, in any current or future media, including reprinting/republishing this material for advertising or promotional purposes, creating new collective works, for resale or redistribution to servers or lists, or reuse of any copyrighted component of this work in other works. 


\title{
Image Deblurring for Navigation Systems of Vision Impaired People Using Sensor Fusion Data
}

\author{
Nimali Rajakaruna ${ }^{1}$, Chamila Rathnayake ${ }^{2}$, Kit Yan $\mathrm{Chan}^{3}$, Iain Murray ${ }^{4}$ \\ Department of Electrical and Computer Engineering \\ Curtin University \\ Perth, Western Australia \\ nimali.rajakaruna@curtin.edu.au1, r.m.rathnayake@postgrad.curtin.edu.au², kit.chan@curtin.edu.au ${ }^{3}$, I.Murray@curtin.edu.au ${ }^{4}$
}

\begin{abstract}
Image deblurring is a key component in vision based indoor/outdoor navigation systems, as blurring is one of the main causes of poor image quality. When images with poor quality are used for analysis, navigation errors are likely to be generated. For navigation systems, blurring is mainly caused by camera movement, as the camera is continuously moving by the body movement. This paper proposes a deblurring methodology that takes advantage of the fact that most smart- phones are equipped with 3-axis accelerometers and gyroscopes. It uses data of the accelerometer and gyroscope to derive a motion vector calculated from the motion of the smartphone during the imagecapturing period. A heuristic method, namely particle swarm optimization, is developed to determine the optimal motion vector, in order to deblur the captured image by reversing the effect of motion. Experimental results indicated that deblurring can be successfully performed using the optimal motion vector and that the deblurred images can be used as a readily approach to object and path identification in vision based navigation systems, especially for blind and vision impaired indoor/outdoor navigation. Also, the performance of proposed method is compared with the commonly used deblurring methods. Better results in term of image quality can be achieved.
\end{abstract}

Keywords - image deblurring; inertial sensors; vision impaired navigation; particle swarm optimization

\section{INTRODUCTION}

Image blurriness is one of the primary causes of poor image quality in image acquisition and can significantly degrade the structure of sharp images. Atmospheric turbulences, out-offocus and the motion of camera or scene would cause the blur. One of the most common reasons for image discarding is camera shaken blur when the light conditions are poor. The camera shake is high in human way finding application as the movement of the body causes shake in the camera. Although faster shutter speeds would reduce the motion blur that can increase camera noise and availability of high-speed cameras in mobile phones and embedded systems is very low.

Real time object detection and path identification using edge detection is a main part of image processing in way finding systems for vision-impaired people [1]. Low-resolution images are used for this to reduce the complexity and computational demands as way finding systems have to be implemented as an embedded system. The discontinuities of detected edges can be increased if the edges are detected with the bluriness of the image. When too many unnecessary discontinuities are generated, errors for object detection or path identification are likely to be produced, thereby creating potential dangers for the vision-impaired people if errors exist in the navigation system.

In fact, the approaches for image deburring can be divided into two categories namely blind deconvolution and non-blind deconvolution. Blind deconvolution is used to deblur the image when information for the camera motion is not available. Nonblind deconvolution can be used when the point-spread function (PSF) is known. As the PSF is an unknown in the conventional image capturing applications, the PSF is unknown and hence the derivation of the solution is more difficult.

Recent technologies of mobile devices enable the estimation of the PSF using the embedded inertial sensors. While the 3-axis accelerometer gives the linear motion the 3axis gyroscope gives the rotary motion. However, the main challenge in using the accelerometer to compute motion is the noise accumulation when performing integration of the accelerometer signal to compute velocity and displacement [2], [3]. Also, an appropriate PSF for effective deblurring is difficult to be generated, as the exposure time is mostly short. Hence, it reduces the deblurring performance when an inappropriate PSF is used.

In this paper, a deblurring methodology is proposed by incorporating with the PSF, where the PSF is determined based on the 3-dimentional linear motion of the scene with respect to the camera. A heuristic method namely particle swarm optimization [18] is developed to determine the optimal the parameters of the PSF, in oder to futher improve the deblurring performance. Experimental results show that the proposed method can improve the image quality of the deblurred images. Also, significant improvement can be achieved when comparing with the commonly used deblurring methods [10], including blind decovolution, Wiener filter, Lucy-Richardson method and the regularized filter.

In Section II, previous work related to image deblurring with and without using inertial sensor data are discussed. Section III describes the proposed deblurring method. Section IV presents the PSF determined by the proposed method and presents the results obtained by proposed method and other commonly used deblurring methods. Comparison with the proposed method and the other tested methods are also given. The conclusion and future work are given in Section V. 


\section{RELATED WORK}

Image de-blurring has recently received significant attention and long lasting problem in the image processing and computer vision fields. Image deblurring can be classified into two types, blind and non-blind deconvolution. Deblurring is more difficult and ill-posed problem when the blur kernel is unknown. When the blur kernel is known all practical solution can be strong than the prior information is unknown about the kernel. Image deblurring is the combination of point spread function (PSF) and non-blind deconvolution. For further literature in this area, we refer the survey article by Kundar and Hatzinakos [4].

The majority of the approaches carried out in deblurring requires minimum of two images of the same scene. RavAcha and Peleg [5] used the information in two motion blurred images, while Lu et al. [6] use a pair of images, one blurry and one noisy, to facilitate capture in low light conditions. But capturing two images in the same scene is not suitable in the area of way finding due to the real time constraints.

Fergus et al. [7] discuss a method on removing camera shake using a single image. This solution identifies the camera motion using an initial kernel estimation, which requires a region without saturation effects. Qi et al. [8] propose a method using a unified probabilistic model of both blur kernel estimation and de-blurred image restoration. Both these methods require complex computational processing which is not suitable for devices with limited processor and memory resources.

Many devices, such as modern smart phones have in-built inertial sensors: gyroscopes and accelerometers. The use of inertial measurement unit (IMU) data to calculate the camera motion may be simpler than the above methods and some of the research already carried out in this area [9], [10], [11], [12]. Hyeoungho et al. [9] proposed a de-blurring method using an integrated depth sensor and IMU sensor with the camera. The joint analysis of these three types of data is used for the better recovery of the real camera motion during the exposure time. This method requires Mathlab scripts to run on a laptop to control the depth sensor and it cannot be applicable in the area of way finding. Horstmeyer et al. [10], Feng et al. [11] and Joshi et al. [12] discuss de-blurring methods using the accelerometer and gyroscopic sensor data and have used DSLR camera, which is more expensive, and a set of sensors, and used an offline computer for image deblurring process.

Sanketi et al. [13] describe methods of anti-blur feedback for visually impaired users of Smartphone camera using the IMU sensor data. Primarily, this feedback is used for the camera stabilization and this is also another use of synchronized IMU data, which is useful in the area of image acquisition. And similar to our work is that of Šindelár ${ }^{\prime}$ and Šroubek [14], that use a smart phone with gyroscope to remove the camera shake blur. In our work we are using gyroscope and accelerometer measure the motion blur because there can be linear motion a well as rotation.
Our research work based on way finding for vision-impair people. The expected frame rate is in the order of 3-5 frames/s as the targeted walking speeds are in the order $100 \mathrm{steps} / \mathrm{m}$ It is expected that captured images will be of poor quality $d$ to lighting conditions, blur and shadows. Image deblurring is a most important role in our research project because we are working on edge detection techniques to detect paths, stairs ways, movable and immovable objects.

\section{Proposed Mthodology}

\section{A. Camera Motion Blur}

In general photography scenarios, the user tries to keep the camera as steady as possible. In this case the motion blur is minimal and most of debluring techniques are targeting this kind of motion blur. However, in way finding applications, there is no control on the movement of the camera, and the result is a heavy motion blur. Therefore, knowing the camera motion is important so that it can be given as an input to the deblurring algorithm.

\section{B. Inertial Sensors and Calibrations}

The sensors used to estimate the movement of the camera are the accelerometer and the gyroscope. Most of high-end smartphones are equipped with a 3-axix accelerometer and a 3 -axis gyroscope that can be used to measure the linear and rotary motions of the phone consecutively. However, the issues in using the accelerometer to compute the linear displacement are the gravity component present in accelerometer data [2], the drift caused by the double integral [3] and the fact that the initial velocity is unknown. Further, when the gyroscope also causes a drift when integrated to compute the angular displacement [3]. However, experiments have indicated that, for a period of $200 \mathrm{~ms}$, the drift caused by the accelerometer integration is in the range of $0.1 \mathrm{~mm}$ once the static error is removed and proper filtering is used and the drift in the angular displacement is in the range of $1 / 1000$. $200 \mathrm{~ms}$ was considered as the exposure time of an image is generally below $100 \mathrm{~ms}$ [15]. Fig. 1 shows the linear and angular displacements computed from the accelerometer and gyroscope sensor data collected while the phone is kept stationary on top of a table.

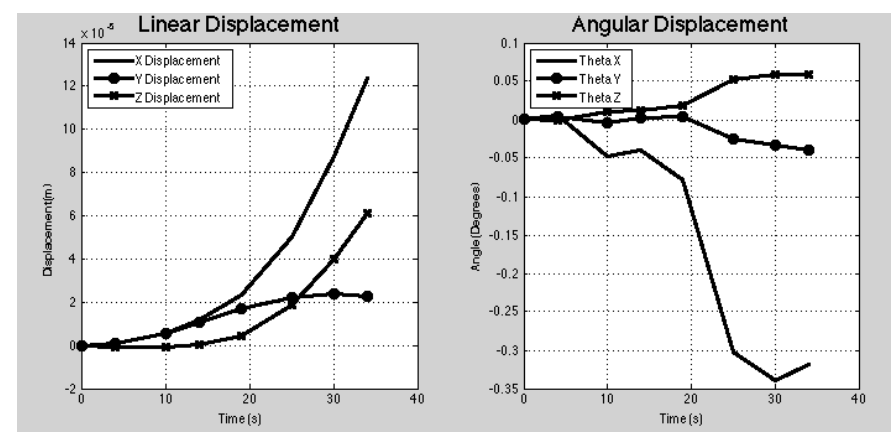

Fig. 1. Error in linear and angular displacements caused by the integration of sensor noise 
This indicates that once proper filtering is done to the sensor signals, the error caused by the drift can be neglected in the scenario we consider.

\section{Determination of PSF}

The blurriness of an image may cause due to linear and angular movements of the camera with respect to the scene. If the camera is considered to be stationary, the scene can be considered as moving with respect to the camera. The blur function or the PSF will both on plane of the scene and perpendicular to that. The component on the plane of the scene causes a linear blur while the perpendicular component causes a zooming effect. Both linear and rotary movements contribute to all these PSF components. Fig. 2 illustrates the placement of the scene w.r.t. the camera, the motion components of the camera and the velocity components of a point on the frame in interest having coordinates $(\mathrm{a}, \mathrm{b})$ w.r.t. the center of the frame. A frame parallel to the plane of the camera that is placed at a distance of 1 is of interest for this analysis. Coordinate system of the camera is taken as the coordinate system for this analysis. Table I shows the motion parameters of the camera and the frame as indicated in the fig. 1 shows the velocity components of the point $(a, b)$. However, contribution of linear velocity components, $V x, V y$ and $V z$ to the velocity of the point on the scene is very small compared to the contribution from angular velocity, 1 can be minimized to 2 . Once the velocity components are computed using 1 or2, 3dimentional displacement components are computed by time integrating each velocity component for the period of exposure using trapezoid rule.

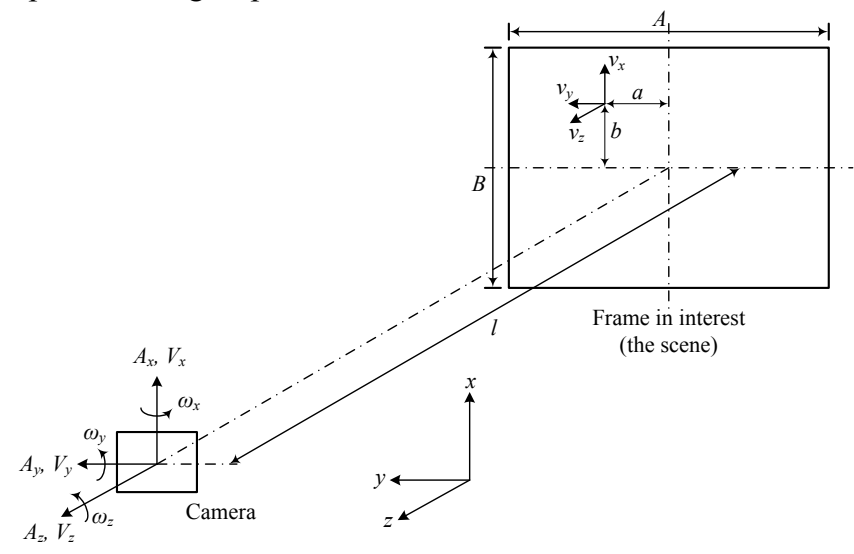

Fig. 2. Illustration of the scene with respect to the camera

Now that the movement of a point within the exposure time is known, it has to be converted to pixel displacements. The challenge faced in this computation was that both sensor size and the focal length were unknown, as the vendors do not provide them.

$$
\begin{aligned}
& v x=-V x+l \omega y+a \omega z \\
& v y=-V y-l \omega x-b \omega z \\
& v z=-V z-a \omega x+b \omega z
\end{aligned}
$$

$$
\begin{aligned}
& v x=l \omega y+a \omega z \\
& v y=-l \omega x-b \omega z \\
& v z=-a \omega x+b \omega z
\end{aligned}
$$

TABLE I. MOTION PARAMETERS OF THE CAMERA AND THE FRAME

\begin{tabular}{|c|l|}
\hline Parameter & Value \\
\hline$A_{x}, A_{y}, A_{z}$ & Linear acceleration of camera along $x, y$ and $z$ axis \\
\hline$V_{x}, V_{y}, V_{z}$ & Linear velocity of camera along $x, y$ and $z$ axis \\
\hline$\omega_{x}, \omega_{y}, \omega_{z}$ & Angular velocity of camera along $x, y$ and $z$ axis \\
\hline$v_{x}, v_{y}, v_{z}$ & Linear velocity of frame w.r.t. camera along $x, y$ and $z$ axis \\
\hline
\end{tabular}

To overcome this problem, a practical measurement was taken to find the ratio $B / l$. It was also confirmed that $B / A=3 / 4$ during this measurement. The values of $A$ and $B$ at the frame in interest were computed using the ratio $\mathrm{B} / 1$ measured. If the resolution of the image is $m \times n(m: n=4: 3)$ the pixel size at the scene can be computed as $A / m$ or $B / n$. From this, the total pixel displacements in $\mathrm{x}$ and $\mathrm{y}$ directions were computed and those are the dimensions of PSF matrix.

The next task was to compute the PSF matrix from the displacements computed. The challenge here was that the samples of $x$ and $y$ displacements ( $d x$ and $d y$ ) computed placed several columns and rows apart in the PSF matrix. Therefor, linear interpolation was used to fill the gap between those samples. Fig. 3 show the algorithm used to derive the PSF from computed displacements on the plane of the scene. The movement along z-axis was not incorporated in this computation so that the zooming can be considered separately.

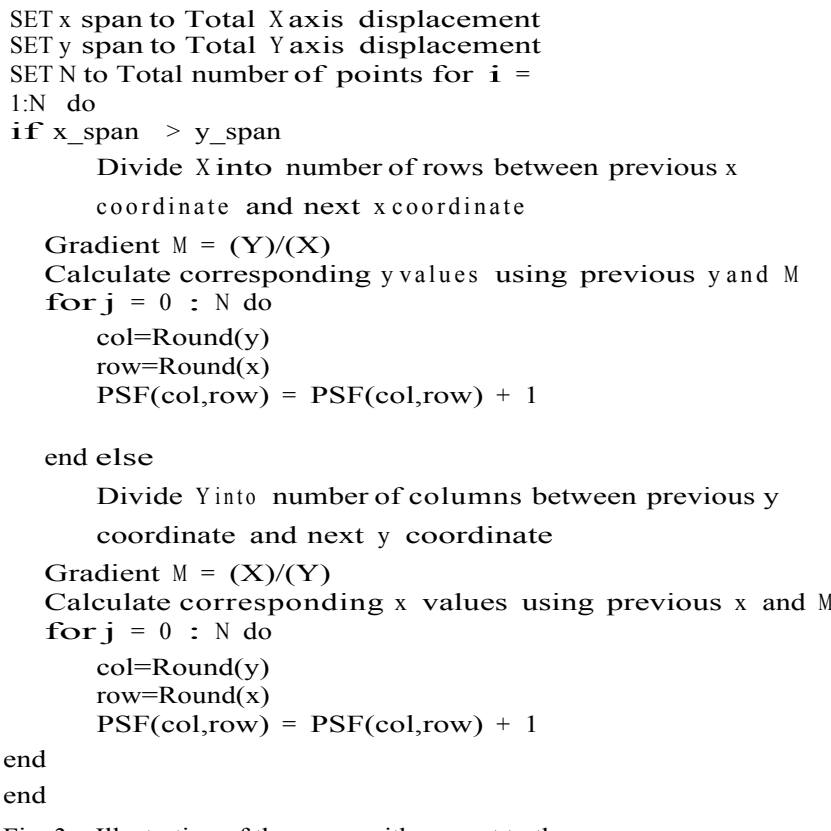

Fig. 3. Illustration of the scene with respect to the camera

When an appropriate PSF is determined, the deblurring can be conducted on the blurred image and the image quality can be improved. To further improve the PSF on deblurring, the 
optimal alignment parameters namely $\mathrm{a}, \mathrm{b}$ and in equations (1) and (2) is necessary to be determined with respect to the image quality. The following section presents a commonly used heuristic method namely particle swarm optimization (PSO)[18] in order to determine the optimal alignment parameters. When the optimal alignment parameters are determined, optimal image quality can be obtained for the deblurred image.

\section{Optimization of PSF}

The particle swarm optimization method (PSO) consists of $N_{s}$ particles, where the position of the $j$-th particle at the $g$-th generation is represented by:

$P_{j}^{g}\left(\sigma_{i}\right)=\left(\kappa_{j, 1}^{g}\left(\sigma_{i}\right), \kappa_{j, 2}^{g}\left(\sigma_{i}\right), \kappa_{j, 3}^{g}\left(\sigma_{i}\right)\right)$,

where $\kappa_{j, 1}^{g}\left(\sigma_{i}\right), \kappa_{j, 2}^{g}\left(\sigma_{i}\right), \kappa_{j, 3}^{g}\left(\sigma_{i}\right)$ are represented by three alignment parameters used in the PSF of the debluring filter namely $a, b$ and $l$ respectively. At the $1-s t$ generation with $g=1$, all $\kappa_{j, k}^{g}\left(\sigma_{i}\right)$, with $k=1,2,3$, are generated randomly within their operational ranges, given as $\kappa_{j, 1}^{g}\left(\sigma_{i}\right) \in\left[a_{\min } . . a_{\max }\right], \kappa_{j, 2}^{g}\left(\sigma_{i}\right) \in\left[b_{\min } . . b_{\max }\right]$ and $\kappa_{j, 3}^{g}\left(\sigma_{i}\right) \in\left[l_{\min } . . l_{\max }\right]$. All $\kappa_{j, k}^{g}\left(\sigma_{i}\right)$, with $k=1,2,3$, are evaluated based on quality of the deblurring image which are determined using the image quality analyzer [19]. When $g>1$, each $P_{j}^{g}\left(\sigma_{i}\right)$ are updated based on its velocity, $v e l_{j, k}^{g}\left(\sigma_{i}\right)$, by the following formulation (4):

$$
\kappa_{j, k}^{g}\left(\sigma_{i}\right)=\kappa_{j, k}^{g-1}\left(\sigma_{i}\right)+v e l_{j, k}^{g}\left(\sigma_{i}\right)
$$

where

$$
\begin{aligned}
\operatorname{vel}_{j, k}^{g}\left(\sigma_{i}\right) & =C\left(v_{j, k}^{g-1}\left(\sigma_{i}\right)+\varphi_{1} \cdot \gamma \cdot\left(\text { pbest }_{j, k}\left(\sigma_{i}\right)-\kappa_{j, k}^{g-1}\left(\sigma_{i}\right)\right)\right. \\
& \left.+\varphi_{2} \cdot \gamma \cdot\left(\operatorname{gbest}_{k}\left(\sigma_{i}\right)-\kappa_{j, k}^{g-1}\left(\sigma_{i}\right)\right)\right)
\end{aligned}
$$

$$
\begin{aligned}
& \operatorname{pbest}_{j}\left(\sigma_{i}\right)=\left[\operatorname{pbest}_{j, 1}\left(\sigma_{i}\right) \text { pbest }_{j, 2}\left(\sigma_{i}\right), \text { pbest }_{j, 3}\left(\sigma_{i}\right)\right], \text { and } \\
& \operatorname{gbest}\left(\sigma_{i}\right)=\left[\operatorname{gbest}_{1}\left(\sigma_{i}\right), \operatorname{gbest}_{2}\left(\sigma_{i}\right), \operatorname{gbest}_{3}\left(\sigma_{i}\right)\right] ;
\end{aligned}
$$

$\operatorname{pbest}_{j}\left(\sigma_{i}\right)$ denotes the best previous position of a particle recorded from the previous generation; gbest $\left(\sigma_{i}\right)$ denotes the position of the best particle among all particles; $\gamma$ denotes a random number in the range of $[0,1] ; w$ is an inertia weight factor; $\phi_{1}$ and $\phi_{2}$ are the acceleration constants [1]; and $C$ denotes the constriction factor, that ensures the PSO converges [2], which is given by:

$$
C=\frac{2}{\left|2-\phi-\sqrt{\phi^{2}-4 \phi}\right|} \text {, with } \phi=\phi_{1}+\phi_{2} \text { and } \phi>4 \text {. }
$$

The PSO utilizes pbest ${ }_{j}\left(\sigma_{i}\right)$ and $\operatorname{gbest}\left(\sigma_{i}\right)$ to modify the current location of all $\kappa_{j, k}^{g}\left(\sigma_{i}\right)$ in order to prevent them from moving in the same direction, but to converge gradually towards pbest $_{i}$ and gbest [3]. To further refine the dynamic of $\kappa_{j, k}^{g}\left(\sigma_{i}\right)$, vel ${ }_{j, k}^{g}\left(\sigma_{i}\right)$ is limited by a value which was set as $10 \%-20 \%$ of its range. This limit is employed to avoid $\kappa_{j, k}^{g}\left(\sigma_{i}\right)$ from flying past good solutions or exploring insufficient local solutions. The searching process of the PSO stops when it converges to the optimal alignment parameters with respect to the image quality defined in [19], where the optimal alignment parameters used in the PSF of the debluring filtersatisfactory filter are denoted as

$$
\bar{\kappa}^{o p t}\left(\sigma_{i}\right)=\left[\kappa_{j, 1}^{o p t}\left(\sigma_{i}\right), \kappa_{j, 2}^{o p t}\left(\sigma_{i}\right), \kappa_{j, 3}^{o p t}\left(\sigma_{i}\right)\right] .
$$

\section{EXPERIMENTAL RESULTS}

The PSF is derived using the method described in "Estimation of PSF" section was used to deblur an image captured with synchronized inertial sensor data while the camera is in motion.

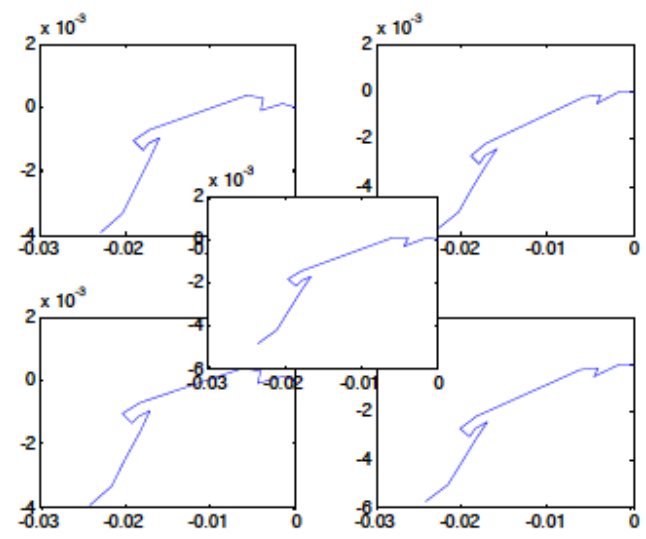

Fig. 4. PSF of different positions of image


Fig. 5. Images with different deblurring techniques. 1: Original image, 2: Blind Deconvolution 3: Wiener filter, 4: Lucy-Richardson 5: Regularized filter, and 6: proposed method (started from the top left hand side to the bottom right hand side)

Fig. 4 shows the PSF estimated using recorded inertial sensor data for the center of the image and for the center of each 
quarter of the image. Fig. 5 shows the original image and deblurred images processed by the proposed method and the five commonly used deblurring methods [10] namely Blind Deconvolution, Wiener filter, Lucy-Richardson method, and regularized filter. The computed PSF was used in the deblurring methods except the blind deconvolution method, as PSF is not required in the blind deconvolution method. The proposed method used the regularized filter as the deblurrer and was engaged with the optimal alignment parameters determined by the PSO discussed in Section III.D.

TABLE II. QUALITY OF THE ORIGINAL AND PROCESSED IMAGE

\begin{tabular}{|l|l|l|}
\hline Image & Quality Score & $\begin{array}{l}\text { Improvement } \\
\text { relatively to the } \\
\text { proposed method } \\
\text { (Percentage) }\end{array}$ \\
\hline Original & 19.8734 & 14.214943 \\
\hline $\begin{array}{l}\text { Blind } \\
\text { deconvolution }\end{array}$ & 19.1981 & 10.333908 \\
\hline Wiener filter & 19.4946 & 12.037931 \\
\hline $\begin{array}{l}\text { Lucy-Richardson } \\
\text { method }\end{array}$ & 19.2360 & 10.551724 \\
\hline Regularized filter & 18.3384 & 5.393103 \\
\hline $\begin{array}{l}\text { The proposed } \\
\text { method }\end{array}$ & 17.4 & Nil \\
\hline
\end{tabular}

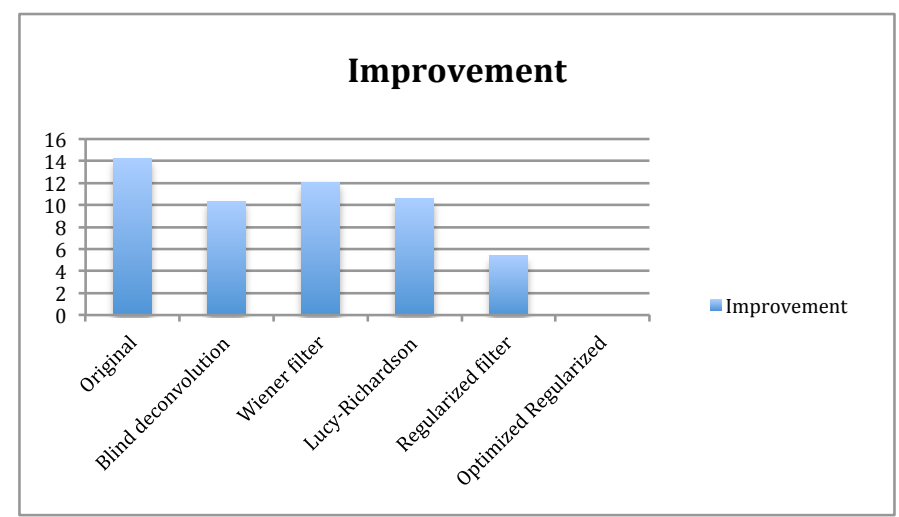

Fig. 6. Improvements in percentage

This experiment was carried out using a Sony Xperia TX smartphone and the images and sensor data were captured while the camera in motion to simulate the navigation. The standard de-blurring and edge detection techniques were applied and tested using MATLAB 2013R. The average computational time for the total processing over the different techniques was measured as 800 milliseconds.

Figure 5 clearly shows that the deblurred images generated by the five deblurred methods are generally better than the original blurred image. However, it is difficult to evaluate the image quality by observing solely the deblurred images. An image quality measure defined by Mittal et al. [19] is used to evaluate the image quality of the deblurred image, where the image quality measure is effective to predict the quality of distorted images with as little prior knowledge of the images or their distortions. Table II shows that quality score evaluated for the original image is the poorest, which is also poorer than those deblurring images. The quality scores obtained by the blind deconvolution method, wiener filter and LucyRichardson method are similar which are poorer than those obtained by the regularized filter and the proposed method. Among the five tested deblurring methods, the proposed method can obtain the best quality score.

To further illustrate the performance of the proposed method, Figure 6 shows the relative improvements when each of the four tested methods, Blind Deconvolution, Wiener filter, Lucy-Richardson method, and regularized filter, is compared with the proposed method, where the relative improvement is the difference between the results obtained by the proposed method and the other tested method, divided by the result obtained by the other tested method. They indicate the relative differences between the results obtained by the proposed method and those obtained by the four tested methods. For the image quality scores, the proposed method obtained improvements with more than $10 \%$ relatively to Blind Deconvolution, Wiener filter, and Lucy-Richardson method. Also, the proposed method obtained an improvement with more than $5 \%$ relatively to the regularized filter. These results further indicate the effectiveness of the proposed method.

\section{CONCLUTION AND FUtURE WORK}

In this paper, a novel method is proposed to determine the optimal PSF using the information from built-in inertial sensors of smartphones, in order to improve the image quality of the deblurred image. A heuristic method namely particle swarm optimization is developed to optimal the parameters of the PSF. Hence, deblurring can be effectively performed using the optimal PSF. Experimental results show that the proposed method can improve the image quality of the deblurred images. Also, significant improvement can be achieved when comparing with the commonly used deblurring filters including blind deconvolution, Wiener filter, Lucy-Richardson method and the regularized filter. The commonly used debluring filters have shown not sufficient performance in debluring images due to motion blur due to body movement, which is commonly seen in vision based navigation systems.

One of the limitations of the proposed method is the sensor accuracy and the noise, which causes to ringing artifacts. This will be a critical factor in edge detection for navigation. The primary focus of this research is way finding for visionimpaired people and hence the noise level should be reduced and the computational cost should be within $100 \mathrm{~ms}$. This will be a topic of our future work which is to introduce an optimized novel algorithm for de-blurring using an inertial sensor data based PSF. 


\section{REFERENCES}

[1] Rajakaruna N., Murray I., "Edge detection for Local Navigation System for Vision Impaired People Using Mobile Devices", International Conference on Mathematical Sciences \& Computer Engineering, Malaysia,

29-30 Nov. 2012.

[2] K. Abhayasinghe and I. Murray. (2012, Nov.). "A novel approach for indoor localization using human gait analysis with gyroscopic data," in Third International Conference on Indoor Positioning and Indoor Navigation (IPIN2012) [Online], Sydney, Australia, 2012. Available: http://www.surveying.unsw.edu.au/ipin2012/proceedings/submissions/ 22_Paper.pdf [Mar. 5, 2013].

[3] D. Sachs. Google Tech Talk, Topic: "Sensor Fusion on Android Devices:

A Revolution in Motion Processing." [On-Line], Aug. 2, 2010. Available: http://www.youtube.com/watch?v=C7JQ7Rpwn2k [Oct. 27, 2011].

[4] Kundur, D.; Hatzinakos, D., "Blind image deconvolution," Signal Processing

Magazine, IEEE, vol.13, no.3, pp.43,64, May 1996

[5] Rav-Acha, A., \& Peleg, S. 2005. Two motion blurred images are better

than one. Pattern Recognition Letters 26, 311-317.

[6] Yuan, Lu, Sun, Jian, Quan, Long, \& Shum Heung-Yeung. (2007). Image

deblurring with blurred/noisy image pairs. Paper presented at the ACM

SIGGRAPH 2007 papers, San Diego, California.

[7] Fergus, Rob, Singh, Barun, Hertzmann, Aaron, Roweis, Sam T., \&

Freeman, William T. (2006). Removing camera shake from a single photograph. Paper presented at the ACM SIGGRAPH 2006 Papers,

Boston, Massachusetts.

[8] Shan, Qi, Jia, Jiaya, \& Agarwala, Aseem. (2008). High-quality motion deblurring from a single image. Paper presented at the ACM SIGGRAPH 2008 papers, Los Angeles, California.

[9] Hyeoungho, Bae, Fowlkes, C. C., \& Chou, P. H. (2013, 15-17 Jan. 2013).

Accurate motion deblurring using camera motion tracking and scene depth. Paper presented at the Applications of Computer Vision (WACV), 2013 IEEE Workshop on

[10] Horstmeyer, Roarke, (2010), Camera Motion Tracking for De-blurring and Identification. MIT Media Lab MAS 863 Final Project.

[11] Feng, JIN, Tian,LI. Digital image stabilization system based on inertial measurement module School of Automation, Beijing Institute of Technology, Beijing 100081, China.

[12] Joshi, Neel, Kang, Sing Bing, Zitnick, C. Lawrence, \& Szeliski, Richard. (2010). Image deblurring using inertial measurement sensors. Paper presented at the ACM SIGGRAPH 2010 papers, Los Angeles, California. [13] Sanketi, Pannag R., \& Coughlan, James M. (2010). Anti-blur feedback for visually impaired users of smartphone cameras. Paper presented at the Proceedings of the 12th international ACM SIGACCESS conference on Computers and accessibility, Orlando, Florida, USA.

[14] Šindelá r $\mathrm{r}$ O, Šroubek F; Image deblurring in smartphone devices using built-in inertial measurement sensors. J. Electron. Imaging. 0001;22(1):011003-011003.

[15] A. Davis, (2012, Apr. 24). "What are the shutter speed and ISO ranges for the iPhone 4S camera?" [Weblog entry]. Ask Different. Available:http://apple.stackexchange.com/questions/49556/what-aretheshutter-speed-and-iso-ranges-for-the-iphone-4s-camera [Mar. 5, 2013].

[16] R.C. Eberhart and Y. Shi, Comparison between genetic algorithms and particle swarm optimization, in Evolutionary Programming VII. New York: Springer-Verlag, LNCS, vol. 1447, pp. 611-616, 1998.

[17] F. Bergh and A.P. Engelbrecht, A study of particle swarm optimization particle trajectories, Information Sciences, vol. 176, no. 8, pp.937-971, 2006.

[18] K.E. Parsopoulos and M.N. Vrahatis, On the computation of all global minimizers through particle swarm optimization, IEEE Transactions on Evolutionary Computation, vol. 8, no. 3, pp. 211-224, 2004.

[19] Mittal, A., Soundararajan, R. \& Bovik, A. C. (2013). Making a "Completely Blind" Image Quality Analyzer. IEEE Signal Process. Lett., 20, 209-212. 